\title{
Kompletter AV-Block bei jungen Patienten: Woran denken und wie abklären?
}

\author{
Gschwend, S ; Keller, D I
}

DOI: https://doi.org/10.1024/1661-8157/a000972

Other titles: Complete AV block in younger patients: what to think of this and how to investigate it?

Posted at the Zurich Open Repository and Archive, University of Zurich

ZORA URL: https://doi.org/10.5167/uzh-67480

Journal Article

Accepted Version

Originally published at:

Gschwend, S; Keller, D I (2012). Kompletter AV-Block bei jungen Patienten: Woran denken und wie abklären? Praxis, 101:819-825.

DOI: https://doi.org/10.1024/1661-8157/a000972 
DOI 10.1024/1661-8157/a000972

((PX_13_Rubrik CME))

\section{Kompletter AV-Block bei jungen Patienten: Woran denken und wie} abklären

Im Artikel verwendete Abkürzungen:

AV-Block

Atrioventrikulärer Block

$\mathrm{BD}$

Blutdruck

HJR Hepatojugulärer Reflux

ICR Intercostalraum

TPPA

Treponema pallidum Partikelagglutinationstest

TSH

Thyreoidea-stimulierendes Hormon (Thyreotropin)

VDRL

Venereal Disease Research Laboratory (Syphilis-Nachweis-Bluttest)

Der atrioventrikuläre Block (AV-Block) wird definiert als Verzögerung oder Unterbruch in der Fortleitung eines elektrischen Impulses vom Vorhof zu den Ventrikeln als Folge eines anatomischen oder funktionellen Problems des Reizleitungssystems. Beim drittgradigen oder kompletten AV-Block (Abb. 1) erreicht kein Vorhofimpuls den Ventrikel, sodass ein unterhalb des Blocks gelegener Schrittmacher einen Ersatzrhythmus generiert. Der Block kann auf Höhe des AV-Knotens oder im infranodalen Reizleitungssystem lokalisiert sein. Anhand der Morphologie des QRS-Komplexes vom Ersatzrhythmus kann die Lokalisation des Blockes abgeschätzt werden:

- schmaler QRS-Komplex = Block im AV-Knoten oder proximalen His-Bündel,

- breiter QRS-Komplex = Block im distalen His-Bündel oder in den Ventrikeln.

Je distaler der Block, umso langsamer der Ersatzrhythmus und umso ausgeprägter die Symptome beim betroffenen Patienten.

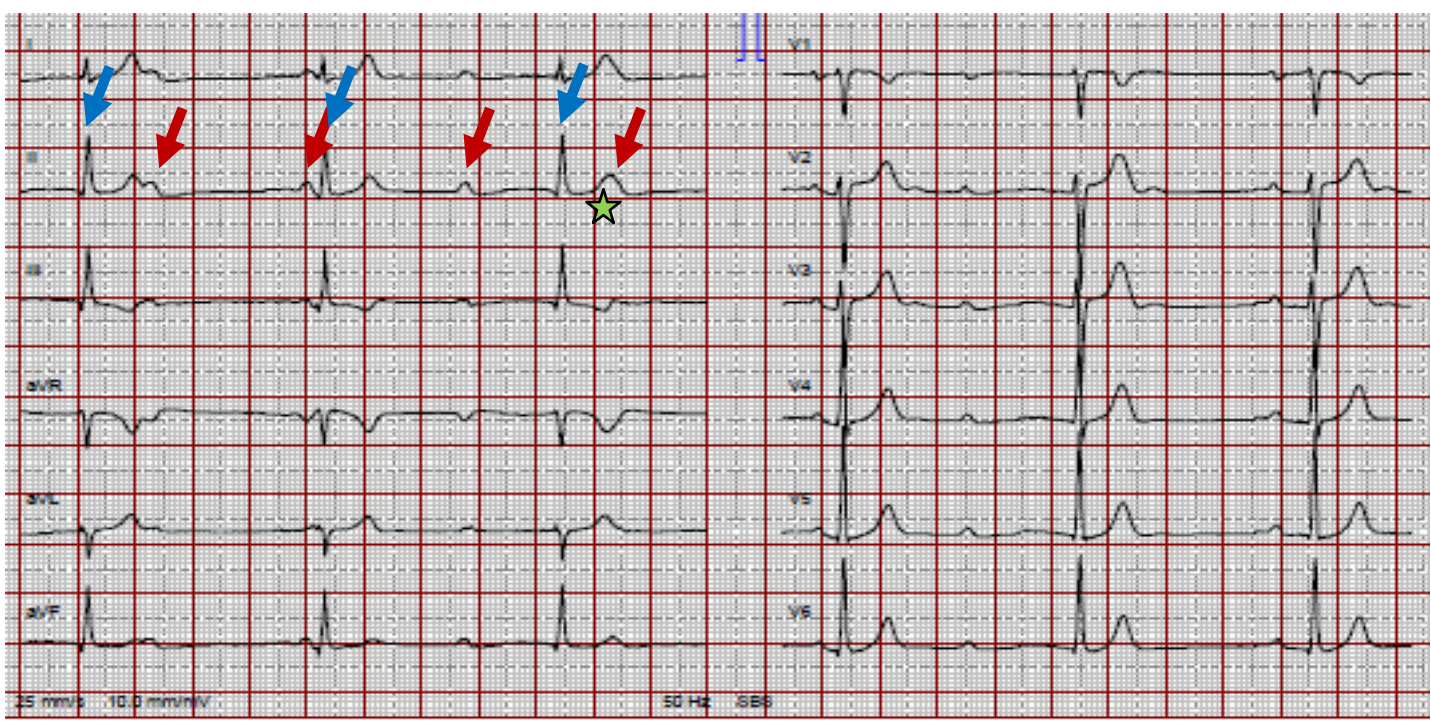

Abb. 1: Beispiel eines kompletten AV-Blocks im 12-Ableitungs-EKG.

Zeichenlegende: $=\mathrm{P}-$ Welle; $=\mathrm{QRS}-$ Komplex, $\boldsymbol{\iota}^{\wedge}=\mathrm{P}-$ Welle in T-Welle.

Klinik ((Ü1)) 
Die Klinik des kompletten AV-Blocks ist abhängig von der Frequenz des Ersatzrhythmus.

Sie kann von Schwindel über Präsynkope und Synkope (Adams-Stokes-Attacken) bis zu ventrikulärer Tachykardie und Kammerflimmern variieren. Die langsame Herzfrequenz kann auch eine vorbestehende Herzinsuffizienz oder Angina Pectoris verstärken.

\section{Generelle Ursachen des kompletten AV-Blocks ((Ü1))}

Bei jungen, bis anhin gesunden Patienten stehen die in Tabelle 1 kursiv gedruckten erworbenen Ätiologien im Vordergrund. Die kongenitalen Formen können sich bereits nach der Geburt manifestieren. Beim seltenen familiären AV-Block können Symptome im Kindesalter auftreten.

Tab.1: Ätiologien des AV-Blocks

\begin{tabular}{|c|c|}
\hline Idiopathisch & Fibrosierung und Sklerosierung des Reizleitungssystems [1] \\
\hline Ischämisch & Akuter Myokardinfarkt, chronische Ischämie \\
\hline Infiltrative Prozesse & $\begin{array}{ll}- & \text { Amyloidose } \\
\text { - } & \text { Sarkoidose [2] } \\
\text { - } & \text { maligne Infiltration (Lymphom, Myelom) }\end{array}$ \\
\hline Infektiös (Myokarditis) & $\begin{array}{ll}\text { - } & \text { Borreliose } \\
\text { - } & \text { Rheumatisches Fieber } \\
\text { - } & \text { Diphtherie } \\
\text { - } & \text { Virale Infekte } \\
\text { - } & \text { Toxoplasmose } \\
\text { - } & \text { Syphilis } \\
\text { - } & \text { Bakterielle Endokarditis }\end{array}$ \\
\hline latrogen & 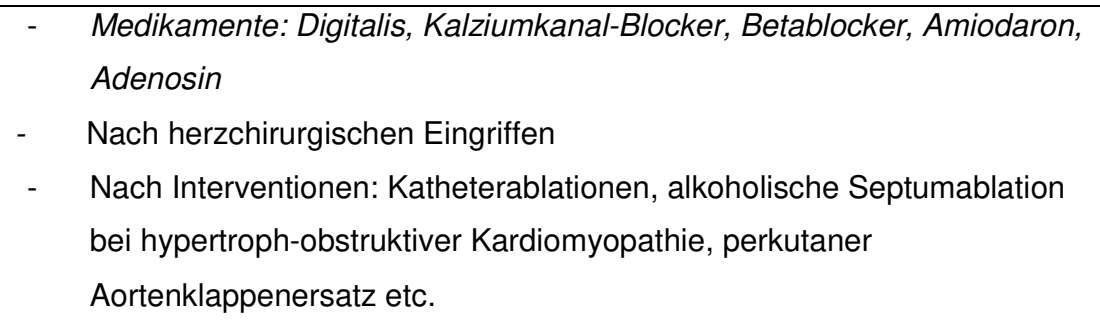 \\
\hline Kongenital & $\begin{array}{ll}\text { - } & \text { Neonatales Lupus-Syndrom (transplanzentäre Passage von anti- } \\
\text { Ro/SSA- oder anti-La/SSB-Antikörper der Mutter) [3] } \\
\text { - } \quad \text { Isolierter AV-Block }\end{array}$ \\
\hline Genetisch & 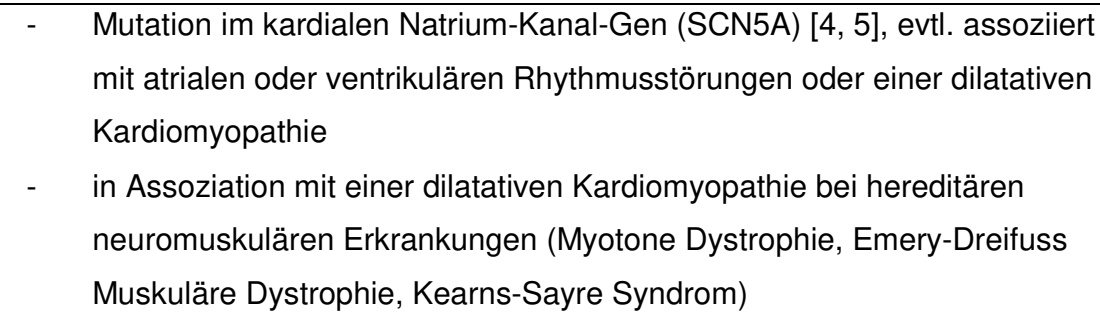 \\
\hline $\begin{array}{l}\text { Andere strukturelle } \\
\text { Herzkrankheiten }\end{array}$ & $\begin{array}{ll}\text { - } & \text { Linksventrikuläre Non-Compaction-Kardiomyopathie } \\
\text { - } & \text { Dilatative Kardiomyopathie, idiopathisch }\end{array}$ \\
\hline Andere & $\begin{array}{ll}\text { - } & \text { Schwere Hyper- oder Hypothyreose } \\
\text { - } & \text { Hyperkaliämie } \\
\text { - } & \text { Trauma }\end{array}$ \\
\hline
\end{tabular}


Abklärungsstrategie bei jungen Patienten mit komplettem AV-Block ((Ü1))

\begin{tabular}{|l|l|}
\hline Anamnese: & - Vorbestehende Kardiopathie \\
& - Zeckenbiss/Erythema migrans erinnerlich \\
& - Medikamentenanamnese \\
& - bekannte Schilddrüsenerkrankung \\
& - Sexualananamnese/Geschlechtskrankheiten \\
& - Familienanamnese \\
& - Symptome eines Infekts (Grippe), B-Symptome \\
& - Herzgeräusch, Zeichen der Herzinsuffizienz \\
& - Integument: Endokarditis-Zeichen, Erythema migrans, Exanthem \\
& - Schilddrüsenpalpation \\
& - Lymphknotenstatus \\
\hline Labor: & - Blutbild, Chemie (Entzündungszeichen, Elektrolyte, TSH) \\
& - Borrelien-Serologie \\
& - Toxoplasmose Antikörper-Bestimmung \\
& - Syphilis-Screening (TPPA als Suchtest, evtl. VDRL) \\
& - bei Fieber Blutkulturen \\
& - ggf. Diphtherie-Antikörper \\
\hline Rx Thorax: & Mediastinale Lymphadenopathie, Herzgrösse \\
\hline Echokardiographie: & Strukturelle Herzkrankheit, Vitien \\
\hline
\end{tabular}

I.

Therapie ((Ü1))

In erster Linie müssen reversible Ursachen eines kompletten AV-Blockes gesucht und behandelt werden. Zudem sollen Medikamente, die die AV-Überleitung bremsen oder blockieren, sofern immer möglich vermieden werden (cave: inklusive Betablocker-haltige Augentropfen). Gegebenenfalls ist die provisorische Schrittmachertherapie notwendig. Bei permanentem, nicht-reversiblem komplettem AV-Block steht die Einlage eines definitiven Herzschrittmachers (Zweikammerschrittmacher) im Vordergrund. 


\section{Fallbericht zu Kompletter AV-Block bei jungen Patienten}

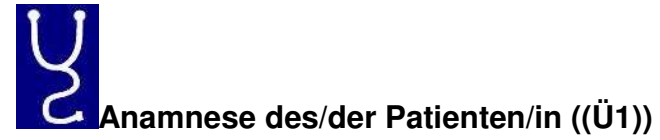

Patient 1: $\widehat{\jmath}$, 20-jährig ((Ü2))

Der Patient berichtet über seit drei Tagen bestehenden

Schwindel mit zweimaliger Bewusstlosigkeit, thorakalem

Druck sowie Mühe beim Atmen. Die Symptomatik habe

plötzlich begonnen. Keine Herzoperation, keine

Herzkrankheiten in der Familie. Im Alltag generell gut

belastbar, keine Infekte. Keine Medikamente.

Patientin 2: $q$, 26-jährig ((Ü2))

Die Patientin berichtet über wiederholte Episoden von kurzzeitiger Bewusstlosigkeit seit zwei Monaten. Nach ein paar Sekunden komme sie jeweils wieder zu sich und sei dabei vollkommen orientiert. Am Vorstellungstag sei es zum ersten Mal beim Arbeiten zu einem solchen Ereignis gekommen, sie sei dabei beim Sitzen mit dem Kopf auf die Schreibtischplatte aufgeschlagen. Kein Urin- oder Stuhlabgang, kein Zungenbiss. Keine sonstigen Erkrankungen bekannt, gute Leistungsfähigkeit im Alltag. Familienanamnese für kardiale Erkrankungen unauffällig. Medikamente: Yasmin ${ }^{\circledR}$ Antibabypille.

\section{Weg zur Diagnose/Kommentar ((Ü1))}

Auffällig sind bei beiden bis anhin gesunden

Patienten wiederholte Synkopen.

Der anhaltende Schwindel sowie thorakale Druck weist beim 20-jährigen Patienten auf eine mögliche kardiale Ursache der Synkopen hin. Beide Patienten haben eine blande persönliche Anamnese und Familienanamnese für kardiale Erkrankungen.

Weg zur Diagnose/Kommentar ((Ü1))

Status ((Ü3))

Patient 1: $\lesssim$ 20-jährig

Guter AZ, BD 118/69 mmHg, Puls 37/min, regelmässig, $\mathrm{SO}_{2}$ $100 \%$. Kardial: bradykarde, normale, rhythmische Herztöne, kein Nebengeräusch, Halsvenen nicht gestaut, HJR negativ, keine Ödeme, Fusspulse bds. gut palpabel. Pulmonale und abdominale Untersuchung regelrecht. Integument unauffällig, keine palpable Lymphadenopathie, Schilddrüse unauffällig.

\section{Patientin 2: $\uparrow$ 26-jährig}

BD 131/66 mmHg, Puls 35/min, regelmässig, $\mathrm{SO}_{2} 100 \%$. Kardial: 1/6 Systolikum 2.-3. ICR rechts ohne Fortleitung, keine Ödeme, Fusspulse beidseits gut palpabel. Pulmonale und abdominale Untersuchung regelrecht. Integument unauffällig, keine palpable Lymphadenopathie, Schilddrüse
Der wegweisende Befund im Status ist bei beiden Patienten die Bradykardie von 37/min resp. 35/min Diese ist regelmässig und beide scheinen trotz Bradykardie stabil und kardiopulmonal kompensiert zu sein.

Hautveränderungen oder Lymphadenopathie bestehen nicht, beide sind afebril. 


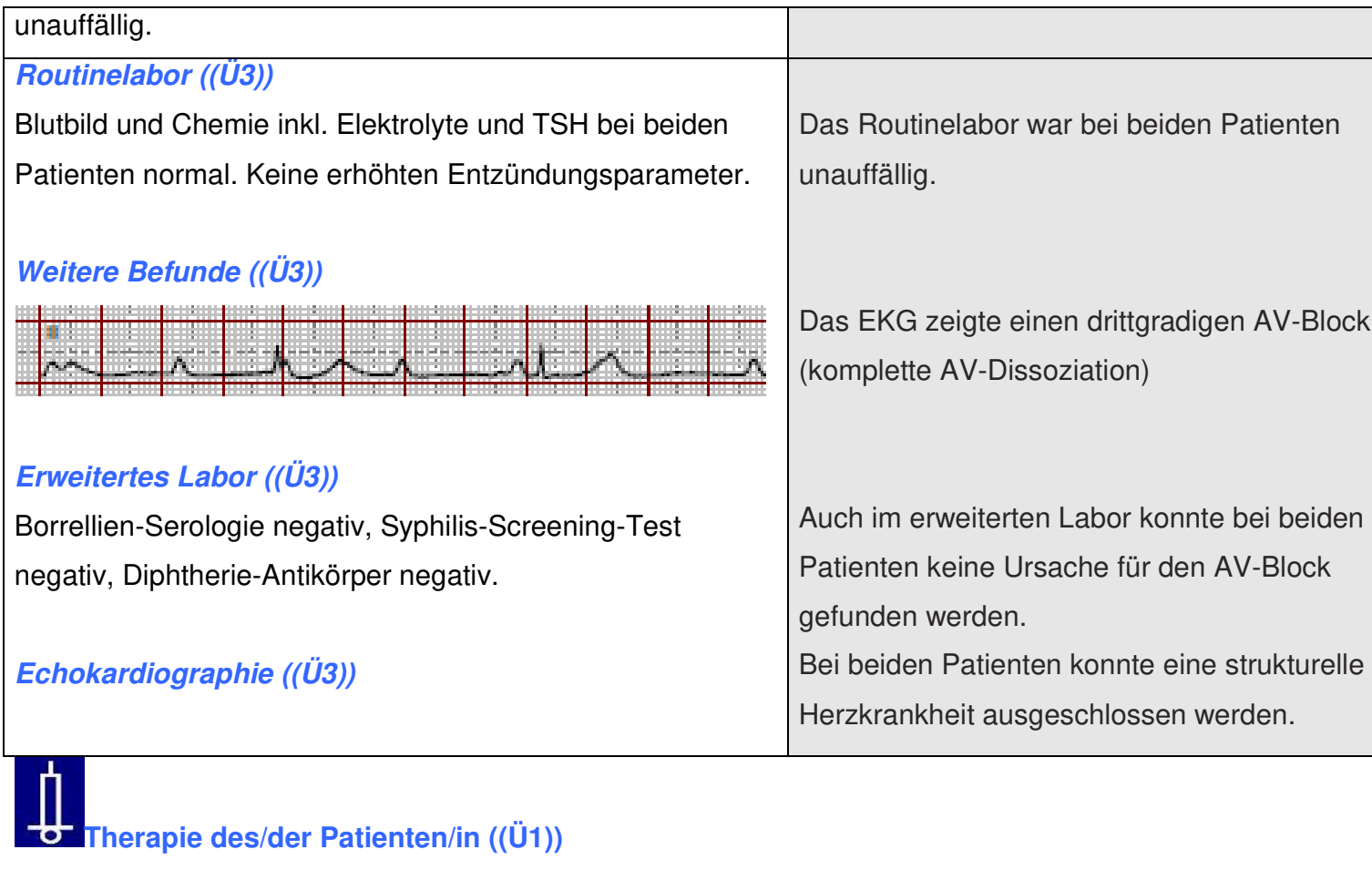

Das Routinelabor war bei beiden Patienten unauffällig.

Auch im erweiterten Labor konnte bei beiden Patienten keine Ursache für den AV-Block gefunden werden.

Bei beiden Patienten konnte eine strukturelle Herzkrankheit ausgeschlossen werden.

\section{if}

herapie des/der Patienten/in ((Ü1))

Da bei beiden Patienten keine reversible Ursache für den kompletten AV-Block gefunden werden konnte und beide trotz Bradykardie stabil waren, wurden sie auf die kardiale Überwachungsstation aufgenommen und je ein Zweikammerschrittmacher (Abb. 2) implantiert. Bei Beschwerdefreiheit konnten sie am ersten postoperativen Tag nach Hause entlassen werden.

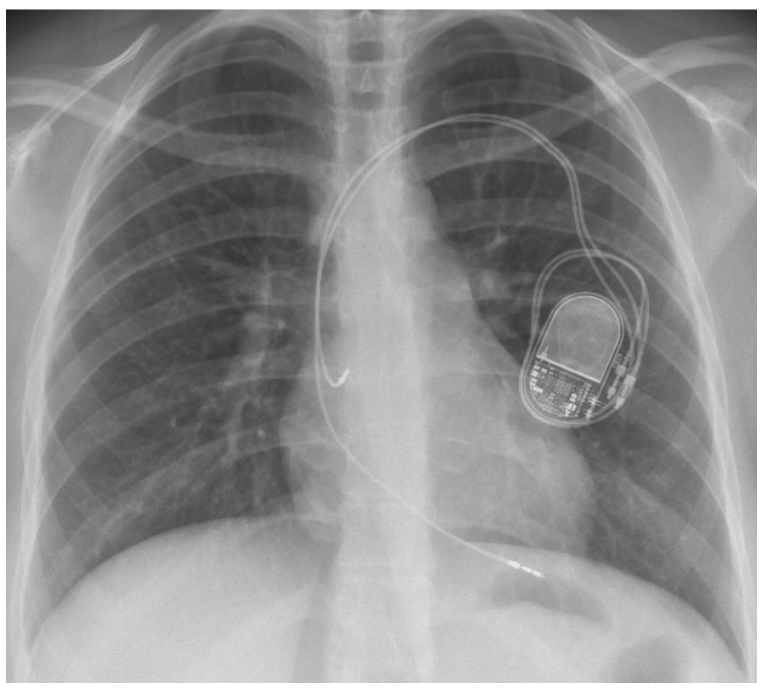

Abb.2: Zweikammerschrittmacher 


\section{Fragen zum kompletten AV-Block bei jungen Patienten}

Frage 1

Welches Blockbild liegt hier vor? (Einfachauswahl, 1 richtige Antwort)

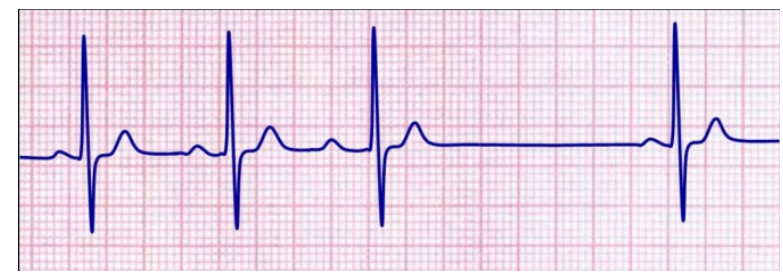
a) AV-Block Grad I
b) AV-Block Grad II, Typ 1 (Wenckebach)
c) AV-Block Grad II, Typ 2 (Mobitz)
d) AV-Block Grad III
e) Kein Blockbild

\section{Frage 2}

An welche Ursachen eines kompletten AV-Blocks soll man bei jungen Patienten mit blander persönlicher und Familien-Anamnese besonders denken? (Einfachauswahl, 1 richtige Antwort)
a) Trauma, familiärer AV-Block, neonatales Lupus-Syndrom
b) Infektiöse Ursachen (Borrellien, viral, Toxoplasmose etc.), Schilddrüsenerkrankungen, Sarkoidose
c) Ischämie, idiopathische Fibrosierung/Sklerosierung, medikamentös-iatrogen
d) Alkohol-Intoxikation, Magnesiummangel, psychogen
e) Amyloidose, Hyperkaliämie, maligne Infiltration

\section{Frage 3}

Welche Ursachen stehen bei älteren Patienten mit komplettem AV-Block im Vordergrund? (Einfachauswahl, 1 richtige Antwort)
a) Trauma, familiärer AV-Block, neonatales Lupus-Syndrom
b) Infektiöse Ursachen (Borrelien, viral, Toxoplasmose etc.), Schilddrüsenerkrankungen, Sarkoidose
c) Ischämie, idiopathische Fibrosierung/Sklerosierung, medikamentös-iatrogen
d) Alkohol-Intoxikation, Magnesiummangel, psychogen
e) Amyloidose, Hyperkaliämie, maligne Infiltration

Frage 4

Ein 25-jähriger, bis anhin gesunder, junger Mann stellt sich mit akut aufgetretener Schwindelsymptomatik sowie zweimaliger Synkope auf der Notfallstation vor. Im Liegen ist er beschwerdearm, sein Blutdruck beträgt 100/60 mmHg, Puls 36/min. Sie diagnostizieren im EKG einen kompletten AV-Block. Wie gehen Sie weiter vor? (Einfachauswahl, 1 richtige Antwort)
a) Ich spritze ihm Atropin und organisiere schnellstmöglich die Einlage eines provisorischen Pacemakers.
b) Der Patient braucht sowieso einen Herzschrittmacher, deshalb rufe ich sofort die Kardiologen zur raschen Planung einer definitiven Pacemaker-Einlage.
c) Ich suche primär nach einer reversiblen Ursache (Anamnese, Labor) und nehme den Patienten auf die Überwachungsstation auf. Solange er im Liegen beschwerdearm und kreislaufstabil ist, kann mit der Schrittmacheranlage zugewartet werden.


d) Ich melde den Patienten für eine dringliche Koronarangiographie an, da die Ischämie die häufigste Ursache für einen kompletten AV-Block ist. Gleichzeitig kann dabei falls nötig auch ein provisorischer Pacemaker eingelegt werden.

e) Da er kreislaufstabil ist, schicke ich inn nach Hause. Junge Patienten erholen sich meist spontan von einem AV-Block.

\section{Frage 5}

Welche Aussage über den kompletten AV-Block stimmt nicht? (Einfachauswahl, 1 richtige Antwort)

a) Je breiter der QRS-Komplex des Ersatzrhythmus, desto tiefer ist die Blockierung im Reizleitungssystems lokalisiert.

b) Ein kompletter AV-Block ist eine häufige Ursache für Synkopen bei jungen Patienten.

c) Beim familiären AV-Block ist es gelungen, Mutationen im kardialen Natrium-Kanal (SCN5A) als Ursache zu detektieren.

d) Vor kardialen Interventionen sowie herzchirurgischen Eingriffen müssen die Patienten über die mögliche Komplikation eines kompletten AV-Blocks aufgeklärt werden.

e) Wenn reversible Ursachen ausgeschlossen sind, ist die Therapie des kompletten AV-Blocks die Einlage eines Herzschrittmachers.

\section{Autoren}

Klinik und Poliklinik für Innere Medizin, Universitätsspital Zürich

Dr. med. Sara Gschwend, PD Dr. med. Dagmar I. Keller,

\section{Korrespondenzadresse}

PD Dr. Dagmar Keller Lang

Uniklinik Balgrist

Paraplegikerzentrum

Forchstrasse 340

$\mathrm{CH}$-8008 Zürich

\section{dagmar.keller@balgrist.ch}

\section{Bibliographie}

1 Davies MJ: Pathology of chronic A-V Block. Acta cardiol 1976; Suppl 21: 19-30.

2 Kandolin R, Lehtonen J, Kupari M: Cardiac sarcoidosis and giant cell myocarditis as causes of atrioventricular block in young and middle-aged adults. Circ Arrhythm Electrophysiol 2011; 4: 303-309.

3 Brucato A, Cimas R, Stramba-Badiale M: Neonatal lupus. Clin Rev Allergy Immunol 2002; 23: 279-299.

4 Schot JJ, Alshinawi C, Kyndt F, Probst V, Hoorntje MT, Hulsbeek M: Cardiac conduction defects associate with mutations in SCN5A. Nat Genet 1999; 23: 20-21.

5 Viswanathan PC, Balser JR: Molecular basis of isolated cardiac conduction disease. Handb Exp Pharmacol 2006; (171): 331-347. 


\section{Antworten zu den Fragen zum kompletten AV-Block bei jungen Patienten aus PRAXIS Nr. 13}

Frage 1

\section{Richtig ist Antwort b).}

Beim AV-Block Grad II Typ Wenckebach verlängert sich die PQ-Zeit zunehmend bis eine Herzaktion vollständig ausfällt.

Ad a): Der erstgradige AV-Block ist definiert als PQ-Zeit $>200$ ms, auf jede P-Welle folgt ein QRS-Komplex.

Ad c): Beim AV-Block Grad II Typ Mobitz fällt in regelmässigen Abständen der QRS-Komplex nach einer PWelle aus, dabei ist die PQ-Zeit nicht verlängert (2:1, 3:1, 4:1-Überleitung)

Ad d): Beim AV-Block Grad III kommt es zu einer kompletten AV-Dissoziation, d.h. Vorhöfe und Kammern kontrahieren unabhängig voneinander.

Frage 2

\section{Richtig ist Antwort b).}

Bei jungen, bis anhin gesunden Patienten stehen infektiöse Ursachen, die zu einer Myokarditis führen können, im Vordergrund. Insbesondere Syphilis, Borreliose sowie Viruserkrankungen sollten gesucht werden. Aber auch an eine Sarkoidose sowie Schilddrüsenerkrankungen (schwere Hypo- und Hyperthyreosen) sollte gedacht werden. Ad a): Ein Trauma, das zu einem drittgradigen AV-Block führt, ist selten. Der familiäre AV-Block manifestiert sich häufig schon im Kindesalter und die Familienanamnese liefert wichtige Hinweise. Das neonatale Lupussyndrom fällt bei der Geburt auf.

Ad c): Eine kardiale Ischämie ist bei jungen Erwachsenen selten, die idiopathische Fibrosierung/Sklerosierung des Reizleitungssystems ist ebenfalls eher als degenerativen Prozess zu verstehen. Die Medikamentenanamnese muss natürlich erfragt werden, wird bei jungen gesunden Patienten aber ebenfalls wenig ergiebig sein.

Ad d): Die akute Alkoholintoxikation ist gehäuft mit atrialen Rhythmusstörungen assoziiert, beim chronischen Alkoholabusus kann es zu Überleitungsstörungen kommen. Magnesiummangel ist nicht mit einem gehäuften Auftreten eines kompletten AV-Blocks assoziiert.

Ad e): Die Amyloidose sowie maligne Infiltrationen sind bei jungen Patienten glücklicherweise selten, auch eine schwere Hyperkaliämie ist bei sonst gesunden Patienten unwahrscheinlich.

\section{Frage 3}

\section{Richtig ist Antwort c).}

Bei älteren Patienten stehen die Ischämie sowie die Degeneration des Reizleitungssystems als Ursache des drittgradigen AV-Blocks deutlich im Vordergrund. Zusätzlich spielen häufig bradykardisierende Medikamente eine wichtige Rolle.

Ad a): Vgl. Frage 2a.

Ad b): Infektiöse Ursachen können auch bei älteren Patienten einen kompletten AV-Block auslösen, sind jedoch seltener als die Ischämie oder die Fibrosierung des Reizleitungssystems.

Dasselbe gilt für die Sarkoidose und die Schilddrüsenerkrankungen. 
Ad d): vgl. Frage 2d.

Ad e): Die Amyloidose, maligne Infiltrationen und schwere Hyperkaliämie können bei älteren Patienten zum kompletten AV-Block führen, sind insgesamt jedoch eher selten.

\section{Frage 4}

\section{Richtig ist Antwort c).}

Im Vordergrund steht bei stabilen Patienten der Ausschluss und ggf. Behebung einer reversiblen Ursache für den AV-Block. Findet sich diese nicht, sind auch junge Patienten auf eine definitive Schrittmachereinlage angewiesen. Ad a): Solange der Blutdruck stabil und der Patient beschwerdearm ist, ist weder die Gabe von Atropin noch die provisorische Schrittmachereinlage nötig.

$A d$ b): Davor sollte bei stabilen Patienten eine potentiell reversible Ursache ausgeschlossen werden.

Ad d): Bei jungen Patienten ist eine Ischämie als Ursache wenig wahrscheinlich, insbesondere wenn keine Thoraxschmerzen bestehen und das EKG keine Ischämiezeichen aufweist.

Ad e): Patienten mit komplettem AV-Block müssen bis zum Beheben des Blocks (durch Behandlung von reversiblen Ursachen oder Schrittmachereinlage) monitorisiert werden.

\section{Frage 5}

\section{Richtig ist Antwort b).}

Der komplette AV-Block führt zwar oft zu Synkopen, bei jungen Erwachsenen sind jedoch neurokardiogene oder vasovagale Synkopen (ca. 50-75\%) viel häufiger als rhythmogene.

Ad a, c, $d, e)$ : Diese Aussagen sind korrekt. 